\title{
From Genetic Testing to Precision Medicine in Epilepsy
}

\author{
Pasquale Striano $^{1,2}$ (D) $\cdot$ Berge A. Minassian ${ }^{3}$
}

Published online: 24 January 2020

(C) The American Society for Experimental NeuroTherapeutics, Inc. 2020

\begin{abstract}
Epilepsy includes a number of medical conditions with recurrent seizures as common denominator. The large number of different syndromes and seizure types as well as the highly variable inter-individual response to the therapies makes management of this condition often challenging. In the last two decades, a genetic etiology has been revealed in more than half of all epilepsies and single gene defects in ion channels or neurotransmitter receptors have been associated with most inherited forms of epilepsy, including some focal and lesional forms as well as specific epileptic developmental encephalopathies. Several genetic tests are now available, including targeted assays up to revolutionary tools that have made sequencing of all coding (whole exome) and noncoding (whole genome) regions of the human genome possible. These recent technological advances have also driven genetic discovery in epilepsy and increased our understanding of the molecular mechanisms of many epileptic disorders, eventually providing targets for precision medicine in some syndromes, such as Dravet syndrome, pyroxidine-dependent epilepsy, and glucose transporter 1 deficiency. However, these examples represent a relatively small subset of all types of epilepsy, and to date, precision medicine in epilepsy has primarily focused on seizure control, and other clinical aspects, such as neurodevelopmental and neuropsychiatric comorbidities, have yet been possible to address. We herein summarize the most recent advances in genetic testing and provide up-to-date approaches for the choice of the correct test for some epileptic disorders and tailored treatments that are already applicable in some monogenic epilepsies. In the next years, the most probably scenario is that epilepsy treatment will be very different from the currently almost empirical approach, eventually with a "precision medicine" approach applicable on a large scale.
\end{abstract}

Keywords Epilepsy $\cdot$ precision medicine $\cdot$ genetic testing $\cdot$ next-generation sequencing $\cdot$ treatment

\section{Introduction}

Epilepsy is a chronic neurological disorder affecting approximately $0.5-1 \%$ of the population worldwide $(\sim 50,000,000$ people) and includes a heterogeneous group of conditions differing in several aspects, including etiology, clinical manifestations, therapeutic approaches, and prognosis $[1,2]$. The main goal of epilepsy treatment is to avoid seizures without

Pasquale Striano

strianop@gmail.com

1 Pediatric Neurology and Muscular Diseases Unit, IRCCS Istituto "G. Gaslini", Genoa, Italy

2 Department of Neurosciences, Rehabilitation, Ophthalmology, Genetics, Maternal and Child Health, University of Genova, Via Gaslini 5, 16148 Genoa, Italy

3 Department of Pediatrics Division of Neurology, University of Texas Southwestern, Dallas, Texas, USA producing detrimental side effects [3]. However, although drug therapy has evolved dramatically in the last two decades, approximately $30 \%$ of patients still experience recurrent seizures, eventually leading to a severe medically and socially disabling condition $[4,5]$.

In the last two decades, advances in genetic techniques, such as oligonucleotide array and the following large-scale studies, have yielded to the identification of the causes of several epilepsy syndromes [6, 7]. Indeed, more than half of all epilepsies have genetic bases and single gene defects in ion channels or neurotransmitter receptors have been associated with several inherited forms of epilepsy, including those previously classified as "idiopathic," but also some focal and lesional forms as well as specific epileptic developmental encephalopathies $[7,8]$.

The term precision medicine aims to describe the treatment of patients with therapy targeted to their specific pathophysiology, in other words, a personalization of treatments that ideally should be targeted towards the precise molecular pathogenesis of disease $[9,10]$. This approach would allow 
physicians to predict more accurately which treatment and prevention strategies for a particular disease will work in specific groups of people. It is in contrast to a "one-size-fits-all" approach, in which treatment is instead developed for the average person, with less or no consideration for differences between individuals [11]. Although examples can be found in several areas of medicine, the role of precision medicine in day-to-day healthcare is relatively limited.

Epilepsy offers an excellent, yet challenging, opportunity for the personalization of treatments, given the very large number of genes discovered - consistent with the fact that, among all organs, the brain utilizes by far the most genes for its development and function [12].

\section{Clinical Assessment: the First Step for a Personalized Approach}

Precision medicine is mainly powered by patient data. Therefore, a careful assessment of the clinical phenotype is the first and crucial step to set up the best approach in a patient with epilepsy. This is mainly based on four cardinal points, namely, family and personal history, clinical evaluation, seizure semiology, and instrumental findings [13]. Family history may indicate the inheritance pattern of familial epilepsies and suggest information about the genetic risk in relatives [9, 14]. Special attention should be paid to consanguinity or history of spontaneous abortions and infantile deaths. Personal history should include information on pregnancy and delivery as well as evaluation of associated neurological comorbidities. In particular, psychomotor development should be accurately evaluated, especially with regard to motor and language skills. In case of intellectual disability, it is essential to establish if this impairment started (global developmental delay/ psychomotor regression) or worsened by the time seizures manifested. Moreover, additional neuropsychiatric features, such as attention deficit hyperactivity disorder, autistic spectrum disorders, and behavioral abnormalities, should be interrogated $[4,6]$.

Physical examination should be focused on identifying facial dysmorphic features, associated abnormalities of the trunk and limbs, or any involvement of other organ dysfunctions or non-neurological involvement. For instance, the presence of café au lait spots or hyperpigmented lesions may suggest the diagnosis of neurofibromatosis type 1 , whereas the identification of hypopigmented macules (ash-leaf spots) and facial angiomatoses may lead to the strong suspect of tuberous sclerosis complex [15]. Although the mechanisms underlying the increased predisposition to brain hyperexcitability differ between these disorders, the genetic and molecular pathways overlap and the mechanistic target of rapamycin (mTOR) signaling cascade is a potential routes for target-specific treatments (see below).
Information about epilepsy features should be as detailed as possible, including detailed description of seizure semiology, age of onset, seizure frequency, and triggering factors or pattern of occurrence. These data should be matched with instrumental findings, in particular ictal or interictal EEG recordings data $[14,16]$. Brain MRI is essential to identify cortical migration defects, vascular abnormalities, and defects of the corpus callosum and cerebellum [16]. In certain conditions, MRI findings alone may help in the choice of the best genetic test, e.g., bilateral periventricular nodular heterotopias in a girl supporting the search of Filamin A mutations through gene panel testing for neuronal migration defects [16].

\section{Targeted Genetic Testing in Epilepsy}

Genetic abnormalities cause or influence more than $70 \%$ of epileptic syndromes [6]. Therefore, genetic testing has become an integral part of pediatric epilepsy work-up. The highest yield group of patients' candidate for genetic testing includes subjects with refractory epilepsy or accompanying dysmorphism, developmental delay/cognitive regression, and intellectual disability.

A variety of genetic tests are currently available to investigate the cause of epileptic disorders [6, 8]. Indeed, the evolution of diagnostic techniques to massively parallel sequencing has allowed a rapid evolution from narrowly applicable tools (e.g., FISH, and single gene testing) to multigene panels, clinical exome sequencing, clinical genome sequencing, and chromosomal microarrays for testing infants and children with epilepsy. Each of these tests should be chosen based on its limitations and expected advantages in the diagnosis of specific epilepsy syndrome and a peculiar clinical phenotype. For instance, cytogenetic studies are particularly helpful in patients with epilepsy and intellectual disability, especially when dysmorphic features coexist or a definite epilepsy syndrome is not recognizable [6-8].

Genomic microarrays are usually the first-tier cytogenetic diagnostic test for patients with associated developmental delay/intellectual disability, autism spectrum disorders and/or multiple congenital anomalies $[6,8,17]$. Array comparative genomic hybridization (array-CGH) is particularly suitable to detect DNA copy number variants (CNVs), such as deletions or duplications [17]. Karyotyping is rather indicated in the search for chromosomal rearrangements that are not detectable by array-CGH, including translocations or complex rearrangements, such as duplication/inversion $15 \mathrm{q} 11$ or ring 20 [18]. Single nucleotide polymorphism (SNP) arrays are particularly useful to reveal regions of homozygosity in the genome and eventually lead to identification of uniparental disomy and parental consanguinity in addition to CNVs. Identification of parental consanguinity in such cases can be important for further diagnostic testing and genetic counseling 
as it is associated with an increased risk of birth defects and autosomal recessive disorders [19].

Altogether, these techniques allow a diagnosis in approximately $10 \%$ of infantile epilepsies and $5 \%$ of epileptic developmental encephalopathies $[6,8]$ as most of epilepsies are instead associated with mutations in single genes. Traditional Sanger sequencing, which allow direct determination of the nucleotide sequence of the exons of a single gene, has been replaced by next generation sequencing (NGS), a microchip-based technology allowing simultaneous sequencing of many genes at relatively low costs [20]. The advent of NGS has made feasible the simultaneous sequencing of hundreds of genes, up to all exons (coding sequences, approximately $1 \%$ of the whole genome) of all human genes through whole-exome sequencing (WES) [21, 22]. Therefore, both gene panel sequencing and WES are being increasingly used in the clinical diagnosis of epileptic disorders with a presumed genetic basis [23, 24].

NGS-based gene panels testing have proven to be particularly useful in the diagnosis of infantile epilepsies, with a diagnostic yield ranging from $20 \%$ up to $50 \%$ compared with the yield of genomic microarrays that is around $5-10 \%[6$, 25]. However, the currently available commercial epilepsy gene panels vary in the number of genes screened, contributing to differences in the diagnostic yield and clinical significance of the test performed [reviewed in 6]. Depending on the type of panel and the characteristics of the investigated population, the diagnostic yield of gene panels is around 30\% (ranging from 10 to $50 \%$ ). However, the limited number of genes that can be sequenced for each panel and the need for a continuous updating of the included genes represents major limitations that have been brilliantly overcome by WES and WGS. Furthermore, the use of WES in parent-offspring trios has also helped identify de novo mutations in patients with complex epileptic developmental encephalopathies [25]. The costs of panels, WES, and whole-exome sequencing (WGS) are falling rapidly so that genetic testing may be the next test after imaging. However, although WGS is very powerful, its application in clinical practice is still limited mainly due to technical difficulties resulting from the handling of large amounts of data, and the complexity of variant interpretation in non-coding regions [26]. Moreover, determining the causative role of a gene mutation may be challenging because the same mutation can cause variable phenotypes, and different mutations may cause similar phenotypes [27].

\section{The Impact of Genetic Testing on Personalized Treatment ("Precision Medicine")}

Precision medicine aims to offer customized treatment for a single epileptic syndrome based on the involved gene and the specific molecular alteration [28]. Table 1 lists some examples of targeted treatments for gene mutations in genetic epilepsy syndromes. Finding a treatable cause of epilepsy is clearly the first step for early diagnosis and treatment and it is essential to improve long-term outcome [29]. Moreover, the discovery of the genetic defect behind a specific form of epilepsy might fully or partially explain the positive or negative (paradoxical) responses to specific antiepileptic drugs. Dravet syndrome, caused by loss-of-function mutations in the SCN1A gene encoding the $\alpha 1$ subunit of the sodium channel gene, is characterized by prolonged fever-related seizures around the age of 6 months, which evolve into a severe developmental encephalopathy with multiple febrile and afebrile seizure types. In this syndrome, clinical worsening may occur with treatment with sodium channel blockers such as carbamazepine or phenytoin, which should be therefore avoided [30, 31]. Conversely, the use of sodium channel blocking is the main therapeutic strategy in patients with developmental epileptic encephalopathy associated with mutations in $S C N 2 A$ and $S C N 8 A$ [32-34], encoding for the $\alpha 2$ and $\alpha 8$ subunits of the neuronal voltage-gated sodium channel [35], in particular patients with the severest epileptic phenotypes, who have been shown to harbor gain-of-function mutations [36, 37]. Another good example for precision medicine in epilepsy is the use of retigabine (ezogabine), a positive allosteric modulator of KCNQ2-5 (Kv7.2-7.5) ion channels, in KCNQ2-associated encephalopathy [38]. Retigabine is the first neuronal potassium channel opener drug used for epilepsy treatment. In vitro studies show that this drug is able to open Kv7 potassium channels thus restoring normal channel function of KCNQ2 encephalopathy associated with loss-of-function mutations [39], with good efficacy against refractory seizures if treatment is started early. However, retigabine use has been limited by the warning of serious adverse effects including skin and retinal pigmentation potentially leading to visual loss for long-term treatment regimens [40]. Another potassium channel blocker proposed for potential precision medicine treatment in epilepsy patients is quinidine. This is an antimalarial and antiarrhythmic drug with a specific inhibitory effect on $K C N T 1$, stabilizing the neuronal membrane by inhibiting the $\mathrm{Na}$ influx required for the initiation and conduction of impulses [41]. Functional studies have shown that quinidine might counteract and reverse the dysfunction of KCNT1-activating mutations in patients with the intractable epilepsy of infancy with migrating focal seizures [42, 43]. However, follow-up studies reported treatment failure in this severe condition, probably due to inability of the drug to reach adequate, effective concentrations in the cerebrospinal fluid. Moreover, dose-limiting cardiac side effects were observed even in the presence of low levels of serum quinidine, suggesting that this drug is likely to be ineffective coupled with considerable cardiac risks [44].

However, the relevance of genetic testing and the eventual implications for treatment go further beyond the role of ion 
Table 1 Examples of precision medicine applied to epilepsy

\begin{tabular}{|c|c|c|c|}
\hline Epilepsy syndrome (\# OMIM) & Gene(s) & Protein function & Possible targeted treatments \\
\hline Pyridoxin-dependent epilepsy (\#266100) & $A L D H 7 A 1$ & Aldehyde dehydrogenase & Pyridoxine (B6 vitamin) \\
\hline $\begin{array}{l}\text { Focal epilepsy with speech disorder, } \\
\text { with/without mental retardation } \\
\text { (\#245570)/EIEE } 27 \text { (\# 616139) }\end{array}$ & GRIN2A, GRIN2B & NMDAR subunits & $\begin{array}{l}\text { NMDAR antagonists (memantine) and } \\
\text { dextromethorphan, potentially useful } \\
\text { (GOF variants) }\end{array}$ \\
\hline EIEE 32 (\# 616366) & KCNA2 & Voltage-gated potassium channel & $\begin{array}{l}\text { Potential efficacy of 4-aminopyridine } \\
\text { (4-AP, Kv1 channels inhibitor) for } \\
\text { GOF variants }\end{array}$ \\
\hline $\begin{array}{l}\text { EIEE } 7 \text { (\#613720); } \\
\text { BFNS1 (\#121200) }\end{array}$ & $K C N Q 2$ & Voltage-gated potassium channel & $\begin{array}{l}\text { Potassium channel openers (Retigabine } \\
\text { and Ezogabine for LOF variants), } \\
\text { potential efficacy of sodium channel } \\
\text { blockers (CBZ) }\end{array}$ \\
\hline $\begin{array}{l}\text { EIEE } 14 \text { (\#614959); Nocturnal } \\
\quad \text { frontal lobe epilepsy (\#615005) }\end{array}$ & KCNT1 & Sodium-activated potassium channel & $\begin{array}{l}\text { Potassium channel openers (Quinidine } \\
\text { for GOF variants) }\end{array}$ \\
\hline PNP oxidase deficiency (\#610090) & $P N P O$ & PNP oxidase & Pyridoxal-5-phosphate \\
\hline $\begin{array}{l}\text { Familial infantile convulsions with } \\
\text { paroxysmal choreoatetosis (\#602066); } \\
\text { BFIS } 2 \text { (\#605751) }\end{array}$ & PRRT2 & Coregulator of synaptic transmission & sodium channel blocker (carbamazepine) \\
\hline Dravet syndrome (\#607208) & SCN1A & Voltage-gated sodium channel subunit & $\begin{array}{l}\text { Avoid sodium channel blockers } \\
\text { (carbamazepine, phenytoin) }\end{array}$ \\
\hline EIEE 11 (\#613721)/BFIS 3 (\#607745) & $S C N 2 A$ & Voltage-gated sodium channel subunit & $\begin{array}{l}\text { Sodium channel blockers for GOF } \\
\text { variants; avoid sodium channel } \\
\text { blockers for LOF variants }\end{array}$ \\
\hline $\begin{array}{l}\text { EIEE } 13 \text { (\#614558); } \\
\text { BFIS } 5 \text { (\#617080) }\end{array}$ & SCN8A & Voltage-gated sodium channel subunit & $\begin{array}{l}\text { Favor sodium channel blockers for } \\
\text { GOF variants }\end{array}$ \\
\hline GLUT1 deficiency (\#606777; \#612126) & $S L C 2 A 1$ & Glucose transporter & Ketogenic diet \\
\hline FCD type II (\#607341) & mTOR, TSC1, TSC2 & mTOR pathway effectors/regulators & Everolimus and other mTOR inhibitors \\
\hline
\end{tabular}

$B F I S$ (benign familial infantile seizures); BFNS (benign familial neonatal seizures); CBZ (carbamazepine); EIEE (early infantile epileptic encephalopathy); FCD (focal cortical dysplasia); GOF (gain-of-function); LOF (loss-of-function); LTG (lamotrigine); NMDAR ( $N$-methyl-D-aspartate receptor); $P H T$ (phenytoin); PNP (pyridoxine 5-prime-phosphate)

channel mutations. For example, epilepsy is a frequent symptom in inborn errors of metabolism and, in some cases, prompt diagnosis may provide the possibility of targeted treatment [45]. The efficacy of the ketogenic diet in the glucose transporter deficiency syndrome is an excellent example of how knowledge of the genetic defect underlying an epileptic disorder may suggest a specific treatment strategy. This condition is caused by mutations in $S L C 2 A 1$ (encoding the glucose transporter GLUT1), which result in deficient glucose transport through the blood-brain barrier [46]. The ketogenic diet is a high-fat diet with low carbohydrate content providing ketone bodies to the brain as an energy source alternative to glucose. Consequently, this dietetic regimen should be effective in conditions with impaired brain glucose supply. Indeed, the appropriate correction of this deficiency results in improvement of both epilepsy and associated neurological symptoms (e.g., movement disorder and intellectual disability) [47]. However, this treatment should be started rapidly, as early treatment significantly improves long-term prognosis in affected children [48]. An additional application of rational therapeutic strategy in genetic metabolic epilepsies is applicable in children with vitamin B6 dependent epilepsy. This is a heterogeneous group of treatable disorders due to mutations in several genes (ALDH7A1, PNPO, ALPL/ALDH4A1, $P R O S C)$, characterized by seizures resistant to anti-seizure drugs but controlled by daily pharmacologic doses of pyridoxine or pyridoxal-5'-phosphate supplementation [49-52]. Finally, the contribution of GATOR complex genes to the etiology of focal epilepsies has recently introduced a novel pathomechanism to a field so far dominated by ion channelopathies [53-55]. The phenotypic and mutational spectrum of epilepsies related to DEPDC5, NPRL2, and NPRL3 genes mainly consists in focal seizures (e.g., hypermotor or frontal lobe seizures), often sleep-related and drug-resistant, sometimes associated with focal cortical dysplasia [56, 57]. The GATOR1 complex is a negative regulator of the mTORC1 pathway, a major signaling cascade regulating cell growth, proliferation, and migration, and represents a potential target for novel therapeutic approaches [58] and has driven the development of mTOR inhibitors in epilepsy, starting with the well-known rapamycin, to several derivative rapalogs including tensirolimus, everolimus, and ridaforolimus [59]. Novel drugs able to block upstream and downstream targets of mTOR pathway (e.g., PF-4708671) [60] are also being 
developed, although further studies are needed to confirm their roles in epilepsy.

\section{Conclusion and Future Perspectives}

Epilepsy is a medical condition defined by recurrent seizures due to excessive electrical discharges in a group of brain cells. Its treatment is limited to a wide range of anti-seizure drugs with different mechanisms of action, which can only provide control of symptoms and are ineffective in about a third of patients or can cause adverse reactions. Thanks to the advances allowed by next-generation sequencing in recent years, the genetic causes of several epileptic conditions have been unveiled and remarkably improved our knowledge on the pathogenesis of epileptogenesis.

Nowadays, genetic tests are available for most developmental epileptic encephalopathies and several idiopathic epilepsies, playing a pivotal role in the diagnosis of these complex conditions. Furthermore, the results of genetic tests may positively influence treatment strategies and, hence, improve patient care. Accordingly, the development of new drugs specifically targeting mutated proteins and selectively addressing pathogenic mechanisms has opened new scenarios for personalized therapeutic approaches (precision medicine). This term summarizes the concept of a personalization of treatments that ideally have to be targeted towards the precise molecular pathogenesis of disease. However, the heterogeneous etiology of epilepsy, the large number of different syndromes and seizure types, together with an individually variable response to pharmacological agents, make the treatment of this condition still challenging. Nevertheless, even in the absence of immediate or direct implications for therapy, a genetic diagnosis can provide relevant information about prognosis, the risk for associated comorbidities, and might also facilitate support from other affected individuals or families. Finally, genetic confirmation may be of paramount importance for counseling and to help with reproductive decisions, including prenatal or preimplantation diagnosis in future pregnancies.

In conclusion, there is still a long way to obtain full integration of precision medicine into clinical practice. In the future, further improvements will probably come from enhancing knowledge of the pharmacogenomics, i.e., the science that studies how these genetic differences affect drug response both in terms of efficacy and susceptibility to adverse drug reactions. Thus, we will probably be able to know the gene variants that alter response to drugs eat pharmacokinetic (e.g., polymorphism in gene involved in drug metabolism) and pharmacodynamic (e.g., polymorphism in brain AED targets, such as ion channels) levels, providing the clinicians valuable tools to predict the efficacy and tolerability of a specific drug in the particular patient. Gene therapy represents a further potentially promising personalized treatment for drug- resistant epilepsy, especially in the context of specific, rare epileptic disorders. In particular, viral-vector-mediated gene transfer offers the opportunity to design a rational treatment that builds on mechanistic understanding of seizure generation and that can be targeted to specific neuronal populations in epileptogenic foci. Nevertheless, the implementation of advanced therapies should be accompanied by the development of advanced tools able to allow clinicians to identify patients suitable for clinical trials or newly approved diseasemodifying therapies early. These include clinical registers and updated databases including information on diseasecausing pathogenic variants, risk variants, modifier genes, polygenic background, and other factors, eventually combined with artificial intelligence algorithms. These ambitious objectives require substantial and implemented collaboration between basic scientists and clinical researchers, including in the development of relevant clinical trial end points and high quality deep phenotyping, eventually leading to translatable, accurate and high-throughput discoveries to obtain precision medicine and improved health outcomes.

Required Author Forms Disclosure forms provided by the authors are available with the online version of this article

\section{References}

1. Striano P. Epilepsy towards the next decade: New trends and hopes in epileptology. Basel: Springer International Publishing, 2015.

2. EpiPM Consortium. A roadmap for precision medicine in the epilepsies. Lancet Neurol 2015;14(12):1219-28.

3. Kwan P, Brodie MJ. Definition of refractory epilepsy: defining the indefinable? Lancet Neurol 2010;9:27-29

4. Symonds JD, Zuberi SM, Johnson MR. Advances in epilepsy gene discovery and implications for epilepsy diagnosis and treatment. Curr Opin Neurol 2017;30:193-199.

5. Kearney H, Byrne S, Cavalleri GL, Delanty N. Tackling Epilepsy With High-definition Precision Medicine: A Review. JAMA Neurol 2019. doi: https://doi.org/10.1001/jamaneurol.2019.2384

6. Dunn P, Albury CL, Maksemous N, et al. Next Generation Sequencing Methods for Diagnosis of Epilepsy Syndromes. Front Genet 2018;9:20

7. Møller RS, Dahl HA, Helbig I. The contribution of next generation sequencing to epilepsy genetics. Expert Rev Mol Diagn 2015;15: $1531-1538$.

8. Orsini A, Zara F, Striano P. Recent advances in epilepsy genetics. Neurosci Lett 2018;667:4-9.

9. Orsini A, Esposito M, Perna D, Bonuccelli A, Peroni A, Striano P. Personalized medicine in epilepsy patients. J Transl Genet Genom 2018;2:16.

10. Striano P, Vari MS, Mazzocchetti C, Verrotti A, Zara F. Management of genetic epilepsies: From empirical treatment to precision medicine. Pharmacol Res 2016;107:426-429.

11. Pitkänen A, Henshall DC, Cross JH, et al. Advancing research toward faster diagnosis, better treatment, and end of stigma in epilepsy. Epilepsia. 2019;60(7):1281-1292.

12. Helbig I, Riggs ER, Barry CA, et al. The ClinGen Epilepsy Gene Curation Expert Panel-Bridging the divide between clinical domain 
knowledge and formal gene curation criteria. Hum Mutat 2018;39(11):1476-1484.

13. Fisher RS, Cross JH, French JA, et al. Operational classification of seizure types by the International League Against Epilepsy: Position Paper of the ILAE Commission for Classification and Terminology. Epilepsia 2017;58:522-530

14. Perry MS, Poduri A. Two Studies, One Message: High Yield of Genetic Testing in Infants and Young Children With Severe Epilepsies. Epilepsy Curr 2018;18:24-26.

15. Stafstrom CE, Staedtke V, Comi AM. Epilepsy Mechanisms in Neurocutaneous Disorders: Tuberous Sclerosis Complex, Neurofibromatosis Type 1, and Sturge-Weber Syndrome. Front Neurol 2017;8:87

16. Bernasconi A, Cendes F, Theodore WH, et al. Recommendations for the use of structural magnetic resonance imaging in the care of patients with epilepsy: A consensus report from the International League Against Epilepsy Neuroimaging. Epilepsia 2019;60:1054 68

17. Coppola A, Cellini E, Stamberger H, et al. Diagnostic implications of genetic copy number variation in epilepsy plus. Epilepsia. 2019;60(4):689-706.

18. Yip MY. Autosomal ring chromosomes in human genetic disorders. Transl Pediatr 2015;4(2):164-74.

19. Fan YS, Ouyang X, Peng J, et al. Frequent detection of parental consanguinity in children with developmental disorders by a combined CGH and SNP microarray. Mol Cytogenet 2013;6(1):38.

20. Hamdan FF, Myers CT, Cossette P, et al. High Rate of Recurrent De Novo Mutations in Developmental and Epileptic Encephalopathies. Am J Hum Genet 2017;101(5):664-685.

21. Berg AT, Coryell J, Saneto RP, et al. Early-Life Epilepsies and the Emerging Role of Genetic Testing. JAMA Pediatr 2017;171(9): 863-871.

22. Monlong J, Girard SL, Meloche C, et al. Global characterization of copy number variants in epilepsy patients from whole genome sequencing. PLoS Genet 2018;14(4):e1007285.

23. Lemke JR, Riesch E, Scheurenbrand T, et al. Targeted next generation sequencing as a diagnostic tool in epileptic disorders. Epilepsia. 2012;53(8):1387-98.

24. Zhang D, Liu X, Deng X. Genetic basis of pediatric epilepsy syndromes. Exp Ther Med 2017;13(5):2129-2133.

25. Epi25 Collaborative. Ultra-Rare Genetic Variation in the Epilepsies: A Whole-Exome Sequencing Study of 17,606 Individuals. Am J Hum Genet 2019;105(2):267-282.

26. Sánchez Fernández I, Loddenkemper T, Gaínza-Lein M, Sheidley BR, Poduri A. Diagnostic yield of genetic tests in epilepsy: A metaanalysis and cost-effectiveness study. Neurology. $2019 \mathrm{https} / / / \mathrm{doi}$. org/10.1212/WNL.0000000000006850

27. El Achkar CM, Olson HE, Poduri A, Pearl PL. The genetics of the epilepsies. Curr Neurol Neurosci Rep 2015;15:39.

28. Myers CT, Mefford HC. Advancing epilepsy genetics in the genomic era. Genome Med 2015;7:91

29. Bowdin S, Gilbert A, Bedoukian E, et al. Recommendations for the integration of genomics into clinical practice. Genet Med 2016;18: $1075-1084$.

30. Wirrell EC, Nabbout R. Recent Advances in the Drug Treatment of Dravet Syndrome. CNS Drugs 2019;33(9):867-881.

31. Striano P, Striano S, Minetti C, Zara F. Refractory, life-threatening status epilepticus in a 3-year-old girl. Lancet Neurol 2008;7(3): 278-84.

32. Wolff M, Johannesen KM, Hedrich UBS, et al. Genetic and phenotypic heterogeneity suggest therapeutic implications in SCN2Arelated disorders. Brain. 2017;140(5):1316-1336.

33. Dilena R, Striano P, Gennaro E, et al. Efficacy of sodium channel blockers in SCN2A early infantile epileptic encephalopathy. Brain and Development 2017;39(4):345-348.
34. Johannesen KM, Gardella E, Encinas AC, et al. The spectrum of intermediate SCN8A-related epilepsy. Epilepsia. 2019;60(5):830 844.

35. Larsen J, Carvill GL, Gardella E, et al. The phenotypic spectrum of SCN8A encephalopathy. Neurology. 2015;84(5):480-9.

36. Reif PS, Tsai MH, Helbig I, Rosenow F, Klein KM. Precision medicine in genetic epilepsies: break of dawn? Expert Rev Neurother 2017r;17(4):381-392.

37. Welzel T, Ziesenitz VC, Waldvogel S, et al. Use of a personalized phenytoin dosing approach to manage difficult to control seizures in an infant with a SCN2A mutation. Eur J Clin Pharmacol 2019;75(5):737-739.

38. Weckhuysen S, Ivanovic V, Hendrickx R, et al. Extending the KCNQ2 encephalopathy spectrum: clinical and neuroimaging findings in 17 patients. Neurology 2013;81(19):1697-703.

39. Millichap JJ, Park KL, Tsuchida T, et al. KCNQ2 encephalopathy: Features, mutational hot spots, and ezogabine treatment of 11 patients. Neurol Genet 2016;2(5):e9.

40. Daniluk J, Cooper JA, Stender M, Kowalczyk A. Survey of Physicians' Understanding of Specific Risks Associated with Retigabine. Drugs Real World Outcomes 2016;3(2):155-163.

41. Dilena R, DiFrancesco JC, Soldovieri MV, et al. Early Treatment with Quinidine in 2 Patients with Epilepsy of Infancy with Migrating Focal Seizures (EIMFS) Due to Gain-of-Function KCNT1 Mutations: Functional Studies, Clinical Responses, and Critical Issues for Personalized Therapy. Neurotherapeutics 2018;15(4):1112-1126.

42. Numis AL, Nair U, Datta AN, et al. Lack of response to quinidine in KCNT1-related neonatal epilepsy. Epilepsia. 2018;59(10):1889 1898.

43. Passey CC, Erramouspe J, Castellanos P, O'Donnell EC, Denton DM. Concurrent Quinidine and Phenobarbital in the Treatment of a Patient with 2 KCNT1 Mutations. Curr Ther Res Clin Exp 2019;90:106-108.

44. Kuchenbuch M, Barcia G, Chemaly N, et al. KCNT1 epilepsy with migrating focal seizures shows a temporal sequence with poor outcome, high mortality and SUDEP. Brain 2019;142(10):2996-3008.

45. Papetti L, Parisi P, Leuzzi V, et al. Metabolic epilepsy: an update. Brain and Development 2013;35(9):827-41.

46. Daci A, Bozalija A, Jashari F, Krasniqi S. Individualizing Treatment Approaches for Epileptic Patients with Glucose Transporter Type1 (GLUT-1) Deficiency. Int J Mol Sci 2018;19(1):E122.

47. Winesett SP, Bessone SK, Kossoff EH. The ketogenic diet in pharmacoresistant childhood epilepsy. Expert Rev Neurother 2015;15(6):621-8.

48. Kass HR, Winesett SP, Bessone SK, Turner Z, Kossoff EH. Use of dietary therapies amongst patients with GLUT1 deficiency syndrome. Seizure 2016;35:83-7.

49. Yeghiazaryan NS, Zara F, Capovilla G, Brigati G, Falsaperla R, Striano P. Pyridoxine-dependent epilepsy: an under-recognised cause of intractable seizures. J Paediatr Child Health 2012;48(3): E113-5.

50. Yeghiazaryan NS, Striano P, Spaccini L, et al. Long-term follow-up in two siblings with pyridoxine-dependent seizures associated with a novel ALDH7A1 mutation. Eur J Paediatr Neurol 2011;15(6): $547-50$.

51. Striano P, Battaglia S, Giordano L, et al. Two novel ALDH7A1 (antiquitin) splicing mutations associated with pyridoxinedependent seizures. Epilepsia. 2009;50(4):933-6.

52. Plecko B, Zweier M, Begemann A, et al. Confirmation of mutations in PROSC as a novel cause of vitamin B 6 -dependent epilepsy. J Med Genet 2017;54(12):809-814.

53. Weckhuysen S, Marsan E, Lambrecq V, et al. Involvement of GATOR complex genes in familial focal epilepsies and focal cortical dysplasia. Epilepsia 2016;57(6):994-1003. 
54. Baldassari S, Picard F, Verbeek NE, et al. The landscape of epilepsy-related GATOR1 variants. Genet Med 2019;21(2):398408.

55. Nieh SE, Sherr EH. Epileptic Encephalopathies: new genes and new pathways. Neurotherapeutics 2014;11:796-806.

56. Sadowski K, Kotulska-Jóźwiak K, Jóźwiak S, et al. Role of mTOR inhibitors in epilepsy treatment. Pharmacol Rep 2015;67:636-646.

57. Dibbens LM, de Vries B, Donatello S, et al. Mutations in DEPDC5 cause familial focal epilepsy with variable foci. Nat Genet 2013;45: $546-551$

58. Mühlebner A, Bongaarts A, Sarnat HB, Scholl T, Aronica E. New insights into a spectrum of developmental malformations related to
mTOR dysregulations: challenges and perspectives. J Anat 2019;235(3):521-542.

59. Ryskalin L, Limanaqi F, Frati A, Busceti CL, Fornai F. mTORRelated Brain Dysfunctions in Neuropsychiatric Disorders. Int J Mol Sci. 2018;19(8):E2226.

60. Sun J, Liu Y, Tran J, O'Neal P, Baudry M, Bi X. mTORC1-S6K1 inhibition or mTORC2 activation improves hippocampal synaptic plasticity and learning in Angelman syndrome mice. Cell Mol Life Sci 2016;73(22):4303-4314.

Publisher's Note Springer Nature remains neutral with regard to jurisdictional claims in published maps and institutional affiliations. 